\title{
Biotransformation of aromatic hydrocarbon: Naphthalene to Aliphatic Hydrocarbons through Staphylococcus pasteuri RD2
}

Ruby Doley and Manoj Barthakur*

Department of Botany, B. Borooah College, Guwahati, Assam, India.

Received: 4/17/2018; Revised: 4/22/2018; Accepted: 4/30/2018

\begin{abstract}
Aromatic hydrocarbons like naphthalene are common environmental pollutants of petrochemical waste. Microorganisms have been exploited since long back to clean up such pollutants by converting them in to either nontoxic or less toxic aromatic or aliphatic compounds. A bacterial strain have been isolated from oil sludge of Guwahati Refinery, Assam and was identified as Staphylococcus pasteuri RD2 (NCBI accession number MG680735) through 16srDNA sequence analysis and molecular phylogeny. The bacterial strain transforms Naphthalene, a common hazardous aromatic hydrocarbon found in petrochemical waste, into a number of less hazardous aliphatic hydrocarbons. Detection of compounds such as Decane, Dodecane, tetradecane, Hexadecane, Eicosane, and heptane by GC-MS analysis of naphthalene enrichment culture broth suggested that the bacterial strain was able to transform naphthalene in to different aliphatic hydrocarbons with less toxicity and having chain length of $\mathrm{C}_{7}$ to $\mathrm{C}_{20}$. It has also been depicted a pathway to obtain aliphatic hydrocarbons with higher caloric value from aromatic hydrocarbon waste.
\end{abstract}

Key words: Biotransformation, Staphylococcus pasteuri, Aromatic hydrocarbon, Naphthalene.

\section{Introduction}

Hydrocarbons are a group of organic compounds composed exclusively of carbon and hydrogen and are either monocyclic- or polycyclic aromatic in structure. Monocyclic aromatic hydrocarbons like benzene, ethylbenzene, toluene and xylene (BTEX) having a single benzene ring, are commonly found in gasoline and are highly volatile substances (Coates et al., 2002). Polycyclic aromatic hydrocarbons (PAHs) contain two or more benzene rings and are relatively less aqueous solubility than monocyclic aromatic hydrocarbons. Aromatic hydrocarbons are considered as highly toxic, and carcinogenic to human health. These pollutants are released from combustion of fossil fuels and hydrocarbons that enter the ecosystem due to their lipophilic property and polluted the environment. The aromatic hydrocarbons like Naphthalene, Benzene, toluene, ethylbenzene, and xylene are commonly found in crude petroleum and petroleum products are considered as one of the major causes of environmental pollution (Farhadian et al., 2008). Among the PAHs naphthalene is the simplest one and has been extensively used as a model for biodegradation. Microbial degradation of aromatic compounds have been reported by a number of authors (Seo et al., 2009, Meckenstock et al., 2004, Lin and Chen, 2010, Mrozik et al., 2003, Annweiler, 2000, Weelink et al., 2002, Doley et al., 2017). Biotransformation of aromatic hydrocarbon in to short and medium chain length polymers (polyhydroxyalconate) have been reported by a number of authors. Hori et al., (2009) and Trautwein et al., (2008) reported the biosynthesis of

\footnotetext{
${ }^{*}$ Corresponding Author:

Dr. Manoj Barthakur,

Associate Professor, Department of Botany

B. Borooah College, Guwahati, Assam, India.

E-mail: barthakurm@yahoo.com
}

short-chain length poly (3-hydroxyalkanoates) by Rhodococcus aetherivorans and Aromatoleum aromaticum from toluene and other volatile aromatic compounds. Ni et al., (2010) reported the biodegradation of BTEX compounds along with the biosynthesis of valuable biopolymers from the aromatic compounds. Pseudomonas putida F1 strain was able to transformed aromatic compounds; benzene, toluene and ethyl benzene (BTE) in to medium chain length hydrocarbons (MCL-PHAs). They further reported the production of elastomeric MCL-PHAs containing 3-hydroxydodecenoate unit from BTE compounds by $P$. fulva TY16, using a novel continuous feeding system of gaseous substrates. The gamma ray mutant strain Psendomonas species EBN8 synthesized medium chain length PHA copolyester as reported by Abid et al., (2016). They identified the metabolite as polyhydroxybutyrate by LCMS and FTIR spectroscopy and exhibited the molecular mass of $\mathrm{m} / \mathrm{z} \quad 448.5$ through ESI-MS analysis. The bioconversion of mono and polycyclicaromatic hydrocarbons in to different chain length polyhydroxyalkanoates have been reported by a number of authors, however, biotransformation of aromatic compounds in to medium or long chain aliphatic compounds have not been reported.

The aim of this study is to convert hazardous aromatic hydrocarbon such as Naphthalene that are common pollutant of petrochemical waste to less hazardous aliphatic hydrocarbons and also to explore a pathway to obtain hydrocarbons with high caloric value from aromatic hydrocarbon waste. 


\section{Materials and Methods}

Chemicals: All chemicals are of analytical grade, purchase from Sisco Research Laboratory Pvt. Ltd., Mumbai.

Bacterial strain: Bacterial strain Staphylococcus pasteuri RD2 has been isolated from oil sludge of Guwahati Refinery, Assam and identified by, 16srDNA sequence analysis and molecular phylogeny as reported earlier (Doley and Barthakur 2017).

Isolation, detection, and identification of metabolite

To identify naphthalene and its transformation products, the bacterial strain was inoculated (CFU $\left.3 \times 10^{6}\right)$ in $250 \mathrm{ml}$ Erlenmeyer flasks containing 100 $\mathrm{ml}$ of naphthalene ( $60 \mathrm{ppm}$ in $\mathrm{n}$-hexane) enrichment nutrient medium and incubated at $37^{\circ} \mathrm{C}$. Seven days after the incubation, the suspension was extracted three times with $\mathrm{n}$-hexane. The combined hexane extract were concentrated in rotary evaporator to about $5 \mathrm{ml}$. Residues were examined for naphthalene and hexane extractable transformation products by GC-MS. Naphthalene enrichment culture without bacterial strain treated as control.

To determine the Naphthalene utilization by the bacterial strain in naphthalene enrichment culture broth was monitored periodically by measuring the optical density at $310 \mathrm{~nm}$ in UV-visible spectrophotometer (Agilent Carry-60 UV-visible spectrophotometer). Culture media inoculated with bacterial suspension without supplementation of naphthalene was treated as control.

\section{Results}

The bacterial strain isolated from oil sludge has already been identified as Staphylococcus pasteuri RD2 through 16srDNA sequence alignment (Clustal W) and molecular phylogeny (Distance Metrix, in MEGA7). The PCR amplicon generated a sequence having 1485 base pair (Fig.1) have been submitted in Gene Bank of NCBI (Accession no. MG680735). Morphology of the bacterial strain was analyzed by Screening Electron Microscopy (Plate 1A and B).

GATGAACGCTGGCGGCGTGCTAATACATG CAAGTCGAGCGAACAGATAAGGAGCTTGC TCCTTTGACGTTAGCGGCGGACGGGTGAG TAACACGTGGATAACCTACCTATAAGACTG GGATAACTTCGGGAAACCGGAGCTAATAC CGGATAAGAT'T'TTGAACCGCATGGT'TCAAT AGTGAAAGACGGCCTTGCTGTCACTTATA GATGGATCCGCGCCGTATTAGCTAGTTGG TAAGGTAACGGCTTACCAAGGCAACGATA CGTAGCCGACCTGAGAGGGTGATCGGCCA CACTGGAACTGAGACACGGTCCAGACTCCT ACGGGAGGCAGCAGTAGGGAATCTTCCGC AATGGGCGAAAGCCTGACGGAGCAACGCC GCGTGAGTGATGAAGGTCTTCGGATCGTA AAACTCTGTTATCAGGGAAGAACAAACGT
GTAAGTAACTGTGCACGTCTTGACGGTAC CTGATCAGAAAGCCACGGCTAACTACGTG CCAGCAGCCGCGGTAATACGTAGGTGGCA AGCGTTATCCGGAATTATTGGGCGTAAAG CGCGCGTAGGCGGTT'TTTTAAGTCTGATG TGAAAGCCCACGGCTCAACCGTGGAGGGT CATTGGAAACTGGAAAACTTGAGTGCAGA AGAGGAAAGTGGAATTCCATGTGTAGCGG TGAAATGCGCAGAGATATGGAGGAACACC AGTGGCGAAGGCGACTTTCTGGTCTGTAA CTGACGCTGATGTGCGAAAGCGTGGGGAT CAAACAGGATTAGATACCCTGGTAGTCCAC GCCGTAAACGATGAGTGCTAAGTGTTAGG GGGTTTCCGCCCCTTAGTGCTGCAGCTAAC GCATTAAGCACTCCGCCTGGGGAGTACGA CCGCAAGGTTGAAACTCAAAGGAATTGAC GGGGACCCGCACAAGCGGTGGAGCATGT GGTTTAATTCGAAGCAACGCGAAGAACCT TACCAAATCTTGACATCCTTTGACCGCTCTA GAGATAGAGTTTTCCCCTTCGGGGGACAA AGTGACAGGTGGTGCATGGTTGTCGTCAG CTCGTGTCGTGAGATGTTGGGTTAAGTCC CGCAACGAGCGCAACCCTTAAGCTTAGTTG CCATCATTAAGTTGGGCACTCTAAGTTGAC TGCCGGTGACAAACCGGAGGAAGGTGGG GATGACGTCAAATCATCATGCCCCTTATGA T'T'TGGGCTACACACGTGCTACAATGGACAA TACAAAGGGCAGCTAAACCGCGAGGTCAA GCAAATCCCATAAAGTTGTTCTCAGTTCGG ATTGTAGTCTGCAACTCGACTACATGAAGC TGGAATCGCTAGTAATCGTAGATCAGCAT GCTACGGTGAATACGTTCCCGGGTCTTGT ACACACCGCCCGTCACACCACGAGAGTTTG TAACACCCGAAGCCGGTGGAGTAACCATT TATGGAGCTAGCCGTCGAAGGTGGGACAA ATGATTGGGGTGAAGTCGTAACA

Fig. 1. Sanger Aligned rDNA sequence of RD2

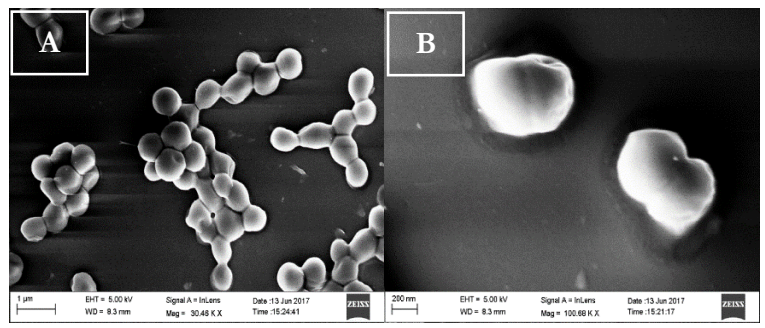

Plate 1. Scaning Electron Microscopic images (magnification $\mathrm{A}=30.46$ and $\mathrm{B}=100.68$ ) of Staphylococcus pasteuri RD2

Biotransformation of Naphthalene by Staphylococcus pasteuri RD2:

The bacterial strain Staphylococcus pasteuri RD2 was able to grow on the culture media by utilizing naphthalene as carbon and energy source. Optical density $\left(\mathrm{OD}_{310}\right)$ of the naphthalene enrichment culture media of Staphylococcus pasteuri RD2 exhibited a gradual decreased from 48 hours onward (fig.2).

GC-MS analysis (Fig. 3) of the biotransformation products of Naphthalene by the bacterial strain 
Staphylococcus pasteuri RD2 revel the presence of heptane (RT 5.357), decane (RT 11.224), Dodecane (RT 17.589), tetradecane (Rt 23.938), Hexadecane (RT 29.300) and Eicosane (RT 33.978) as major products.

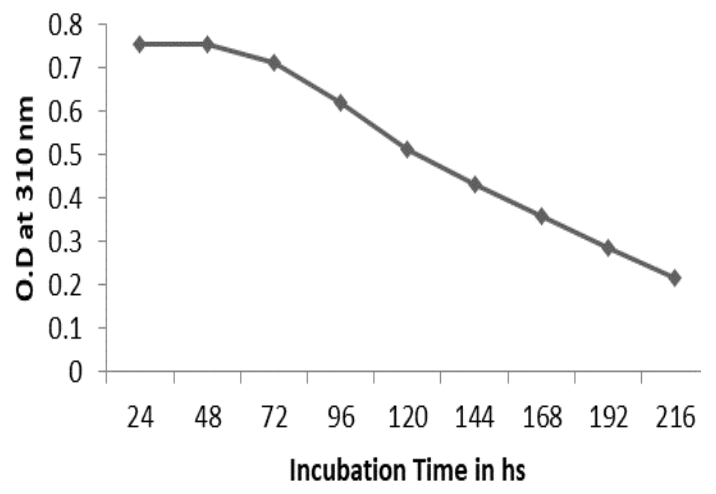

Figure 2. Utilization of naphthalene by Staphylococcus pasteuri RD2

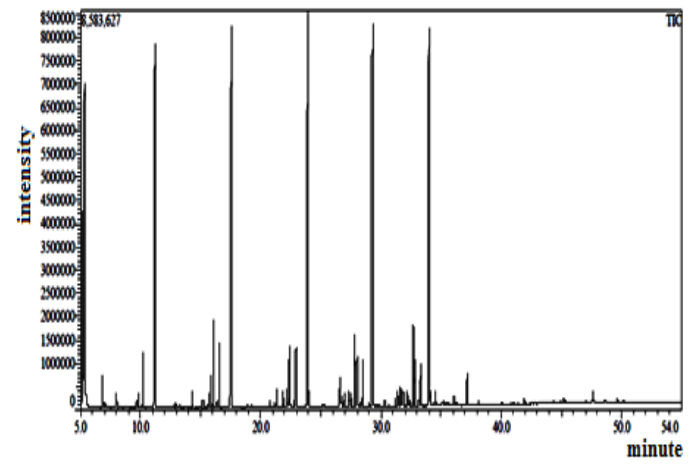

Figure 3. GC-MS chromatogram of naphthalene biotransformation products

\section{Discussion}

The bacterial strain isolated from oil sludge of Guwahati Refinery and was identified as Staphylococcus pasteuri. The DNA sequence of 1485 base pair length (Doley and Barthakur 2017) have been submitted in the NCBI Gene Bank with accession number MG680735(https://www.ncbi.nlm.nih.gov/Taxono my/Browser/wwwtax.cgiiid=45972). Screening Electron Microscopic analysis suggested the coccoid nature of cells, arranged in packets (plate1A, 1B). Gradual decrease of optical density (O.D 310$)$ of naphthalene in the culture broth suggested the utilization of naphthalene by the bacterial strain as carbon and energy source (fig.2).

Microbial degradation of aromatic hydrocarbons has been reported by a number of authors (Karimi et al., 2002, Heider et al., 1999, Abide et al 2016, Doley and Barthakur 2017). We have reported earlier the degradation of naphthalene in to its derivatives by Staphylococcus pasteuri RD2 (Doley and Barthakur (2017). However, limited reports have been found on biotransformation of mono- and polycyclic aromatic compounds in to medium chain length hydrocarbons such as polyhydroxyalcanoate (PHA). Narancic et al., (2012) reported the conversion of a range of polyaromatic hydrocarbon compounds to medium chain length PHA. The bacterial strain Pseudomonas sp. TN301 was able to convert polycyclic aromatic hydrocarbons such as naphthalene, phenanthrene and chrysene in to mcl-PHA. Srivastav and Tripathi (2013) reported the production of medium and short chain length polyhydroxyalkanoate (PHA) by Alcaligenes species MCIM 5085 when supplemented with fatty acid in basal media which could potentially serve as precursors for bioplastic production. Nikodinovic et al., (2008) demonstrated the conversion of monoaromatic compounds such as Benzene, Toluene, Ethylbenzene and Xylene (BTEX) to medium chain length polyhydroxyalcanoate by two Pseudomonas strains strain F1 and mt-2. Pseudomonas putida F1 accumulate mcl-PHA from toluene, benzene and ethylbenzene whereas $P$. putida mt-2 accumulates mcl-PHA from toluene and $p$-xylene. Ward et al., (2005) reported the conversion of aromatic hydrocarbon styrene and phenylacetic acid into polyhydroxyalcanoate by Psendomonas putida CA-3. In this paper we have reported the biotransformation of polycyclic aromatic hydrocarbon naphthalene to aliphatic hydrocarbons by the bacterial strain Staphylococcus pesteuri RD2 isolated from oil sludge of Guwahati Refinery. GC-MS analysis reveals the presence of a number of aliphatic hydrocarbons in naphthalene enrichment culture medium of Staphylococcus pasteuri RD2. Compounds detected such as heptane $\left(\mathrm{C}_{7} \mathrm{H}_{16} \mathrm{O}\right)$, decane $\left(\mathrm{C}_{12} \mathrm{H}_{26}\right)$, Dodecane $\left(\mathrm{C}_{12} \mathrm{H}_{26}\right)$, tetradecane $\left(\mathrm{C}_{14} \mathrm{H}_{30}\right)$, Hexadecane $\left(\mathrm{C}_{16} \mathrm{H}_{34}\right)$ and Eicosane $\left(\mathrm{C}_{20} \mathrm{H}_{42}\right)$ having a chain length of $\mathrm{C}_{7}$ to $\mathrm{C}_{20}$ carbon atom indicated the biotransformation of naphthalene to such aliphatic hydrocarbons. This study reveals that the bacterial strain Staphylococcus pasteuri RD2 have the ability to transform the common pollutant such as naphthalene in to less toxic aliphatic hydrocarbons along with the formation of different carbon length aliphatic hydrocarbons with higher caloric value from the aromatic waste.

\section{Conclusions}

The present studies strongly suggest the ability of Staphylococcus pasteuri RD2 to transform naphthalene into various aliphatic hydrocarbons as major product. The GC-MS analysis detected the probable compounds like Decane, Dodecane, Tetradecane, Hexadecane, Eicosane and Heptane. The bioconversion of Naphthalene in to aliphatic hydrocarbons, mediated by Staphylococcus pasteuri RD2 depict a pathway to manage toxic aromatic hydrocarbon waste released to our environment from different sources as well as it also depict a pathway to obtain aliphatic hydrocarbons with higher caloric value from aromatic hydrocarbon wastes. 


\section{Acknowledgement}

Authors are grateful to University Grant Commission for financial support to the corresponding author to carry out this research work. Authors are highly thankful to IASST, Guwahati for providing SEM and GC-MS facilities. Authors are also thankful to Biotech Hub, and Department of Chemistry, B. Borooah College for providing technical support for DNA isolation and UV-visible spectroscopic facility respectively.

\section{References}

1. Abid S, ZA Raza, A Rehman, T Hussain, Production kinetics of polyhydroxyalkanoates by using Pseudomonas aeruginosa gama ray mutant strain EBN-8 cultured on soybean oil. Biotechnol, 2016, 6: 142-151.

2. Annweiler E, $\mathrm{HH}$ Richnow, G Antranikian, S Hebenbrock, C Garms, S Franke, W Francke, W Michaelis W, Naphthalene degradation and incorporation of Naphthalene-Derived Carbon into Biomass by the Thermophile Bacillus thermoleovorans. Appl Env Microbiol, 2000, 566: 518-532.

3. Coates JD, R Chakraborty, MJ McInerney, Anaerobic benzene biodegradation-a new era. Res Microbiol, 2002, 153:621-628.

4. Doley R, M Barthakur, BS Goswami, Microbial Degradation of Aromatic hydrocarbon: Naphthalene through Nocardiopsis Alba RD3. International J Curr Microbiol App Sc, 2017, 6: 1174-1181

5. Doley R, M Barthakur, Biodegradation of Naphthalene by Staphylococcus pasteuri RD2 Isolated from Oil Contaminated Soil. International J Curr Microbiol App Sc, 2017, 6:1310-1319.

6. Farhadian M, D Duchez, C Vachelard, C Larroche, BTX Removal from Polluted Water through Bioleaching Processes. Appl Biochem Biotechnol, 2008, 151:295-306.

7. Hori K, A Kabayashi, H Ikeda, H Unno, Rhodococcus aetherivorans IARI, a new bacterial strain synthesizing poly (3-hydroxybutyrate-co-hydroxyvalerate) from toluene. (2009). J Biosc and Bioeng, 2009, 107:145150.

8. Heider J, Spormann M Alfred, Beller HR, Widdel F, Anaerobic, bacterial metabolism of hydrocarbons. FEMS Microbiology Reviews, 1999, 22: 459-473.

9. Kathleen Trautwein, KS Simon, LW Wolbrand, TR Halde, K Kuchta, A Steinbuche, R Rabus, Solvent Stress Response of the Denitrifying Bacterium "Aromatoleum aromaticum" Strain EbN1. Appl Env Microbiol, 2008, 74. 2267-2274.

10. Karimi B, M Habibi, M Esvand, Biodegradation of naphthalene using Pseudomonas aeruginosa by up flow anoxic-aerobic continuous flow combined bioreactor. J Environmental health Science \& engineering, 2002, 13:26-36

11. Lin C, ZL Chen, Biodegradation of naphthalene by strain Bacillus fusiformis (FBM). J of Hazardous Materials, 2007, 181:771-777.

12. Meckenstock RU, M Safinowski, C Griebler, Anaerobic degradation of polycyclic aromatic hydrocarbons. FEMS Microbiol Ecol, 2004, 49: 2736.

13. Mrozik A, Z Piotrowska-Seget, K Labuzek, Bacterial degradation and bioremediation of polycyclic aromatic hydrocarbon. Polish J Env Studies, 2003, 12:15-25.

14. Narancic T, ST Kenny, L Djokic, B Vasiljevic, KE O'Connor, J Nikodinovic-Runic, Medium-chainlength polyhydroxyalkanoate production by newly isolated Pseudomonas sp. TN301 from a wide range of polyaromatic and monoaromatic hydrocarbons. J Appl Microbiol, 2012, 113:508-20.

15. Ni YY, MG Chung, SH Lee, HY Park, YH Rhee, Biosynthesis of medium-chain length poly(3hydroxyalkanoatee) by volatile aromatic hydrocarbons-degrading Pseudomonas fulva TY16. Bioresource Tech, 2010, 101:8485-8488.

16. Nikodinovic J, ST Kenny, RP Babu, T Woods, WJ Blau, KE O'Connor, The conversion of BTEX compounds by single and defined mixed cultures to medium-chain-length polyhydroxyalkanoate. Appl Microbiol Biotechnol, 2008, 80:665-673.

17. Srivastava SK, AD Tripathi, Effect of saturated and unsaturated fatty acid supplementation on bio-plastic production under submerged fermentation. Biotech, 2013, 3:389-397.

18. Su-Seo JS, YS Keum, XL Qing, Bacterial Degradation of Aromatic Compounds. Int J Environ Res Public Health, 2009, 6: 278- 309.

19. Ward GP, G de Roo, KE O'Connor, Accumulation of Polyhydroxyalkanoate from Styrene and Phenylacetic Acid by Pseudomonas putida CA-3. Appl Environ Microbiol, 2005, 71:2046-2052.

20. Weelink SAB, HA Miriam, V Eekert, JM Alfons, Degradation of BTEX by anaerobic bacteria: physiology and application. Rev Env Sci Biotechnol, 2010, 9:359-385.

\section{Cite this article as:}

Ruby Doley and Manoj Barthakur. Biotransformation of aromatic hydrocarbon: Naphthalene to Aliphatic Hydrocarbons through Staphylococcus pasteuri RD2. Annals of Plant Sciences 7.5 (2018) pp. 2247-2250.

do http://dx.doi.org/10.21746/aps.2018.7.5.7 